\title{
ERRATUM
}

Fernando Tornos · César Casquet · Carmen Galindo

Francisco Velasco $\cdot$ Angel Canales

\section{A new style of Ni-Cu mineralization related to magmatic breccia pipes in a transpressional magmatic arc, Aguablanca, Spain}

Published online: 20 October 2001

(C) Springer-Verlag 2001

\section{Mineralium Deposita (2001) 36:700-706}

The graphic scale of Fig. 2 (Geological map of the Aguablanca orebody) should be $100 \mathrm{~m}$ instead of $10 \mathrm{~m}$.

The online version of the original article can be found at http://dx.doi.org/10.1007/s001260100204

F. Tornos $(\bowtie)$

Instituto Geológico y Minero de España, Azafranal 48,

37001 Salamanca, Spain

E-mail: ftaitge@iponet.es

C. Casquet · C. Galindo

Departamento Petrologia,

Universidad Complutense de Madrid 28040 Madrid,

Spain

F. Velasco

Departamento Mineralogia y Petrologia,

Universidad Pais Vasco, Apdo 644 Leoia,

48080 Vizcaya, Spain

A. Canales

PRESUR SA, Minas de Cala,

21279 Huelva, Spain 